\title{
ENERGY PROPAGATION IN A CAUCHY ELASTIC MATERIAL*
}

\author{
BY M. A. HAYES** AND R. S. RIVLIN (Lehigh University)
}

1. Introduction. It has been shown by Schouten [1] that in an anisotropic homogeneous elastic material subjected to infinitesimal deformations the energy propagation vector is necessarily normal to the slowness surface. It can be readily shown (see, for example, [2]) that the bicharacteristics are also normal to the slowness surface.

In the present paper, we discuss the relation between the slowness surface and the energy propagation vector in an anisotropic homogeneous Cauchy elastic material (i.e., a material in which the Cauchy stress depends only on the displacement gradients, but for which no strain-energy function exists). The material is assumed to be subjected only to infinitesimal deformations, so that the stress is a linear function of the classical infinitesimal strain components. However, the elastic modulus tensor is not necessarily symmetric, i.e. the Onsager relations are not necessarily satisfied.

We consider the propagation of a second-order discontinuity in such a material and obtain, in Sec. 2, the equation for the slowness surface and for the energy propagation vector. In Sec. 3, we apply these results for a particular choice of the elastic modulus tensor. It is shown that in this case, and a fortiori in more general cases, the energy propagation vector is not normal to the slowness surface. We note that the usual calculation which shows the bicharacteristics to be normal to the slowness surface in an elastic material is still valid in a Cauchy elastic material, so that for such a material the energy propagation vector is not, in general, in the direction of the bicharacteristics.

It must be borne in mind in assessing the physical significance of these results that the physical reality of a Cauchy elastic material is open to question $[3,4]$.

2. The basic equations. We consider a body of Cauchy elastic material to be subjected to infinitesimal deformations. Let $u_{i}$ be the displacement at time $t$ and $\sigma_{i i}$ the stress in a rectangular cartesian coordinate system $x$. Then

$$
\sigma_{i j}=g_{i j k l} u_{k, l},
$$

where $g_{i j k l}$ are constants satisfying the conditions

$$
g_{i j k l}=g_{i i k l}=g_{i j l k}, \quad g_{i j k l} \neq g_{k l i j} .
$$

The latter condition is, of course, not satisfied for an elastic material which possesses a strain-energy function.

In the absence of body forces, the equations of motion may be written

$$
\sigma_{i, i}=\rho \ddot{u}_{i},
$$

where $\rho$ is the density of the material at time $t$ and a dot denotes differentiation with respect to time.

* Received June 5, 1971. The research reported in this paper was supported by a grant from the National Science Foundation to Lehigh University.

** Present address: Department of Mathematics, University of East Anglia, Norwich, England. 
We suppose that a second-order discontinuity propagates in the material and that the surface of discontinuity is

$$
\Phi\left(x_{i}, t\right)=0 .
$$

Then the first spatial and temporal derivatives of $u_{i}$ are continuous everywhere, but the second derivatives undergo a jump on (2.4). We may therefore write $u_{i}$ in the form

$$
u_{i}=b_{i}\left(x_{i}\right) f\left\{\Phi\left(x_{k}, t\right)\right\},
$$

where $b_{i}\left(x_{i}\right)$ is independent of time but possesses continuous first and second spatial derivatives everywhere. $f$ and its first derivative with respect to $\Phi$ are continuous everywhere, while the second derivative of $f$ with respect to $\Phi$ is continuous except on $\Phi=0$, where it undergoes a jump. We thus have, from (2.5),

$$
\left[u_{k, l i}\right]=\left[f^{\prime \prime}\right] b_{k} \Phi_{, l} \Phi_{, i}, \quad\left[\ddot{u}_{i}\right]=\left[f^{\prime \prime}\right] b_{\imath} \dot{\Phi}^{2},
$$

where [ ] denotes the jump and a prime denotes differentiation with respect to $\Phi$.

Introducing (2.1) into $(2.3)$ we see that the jumps $\left[u_{k, l i}\right]$ and $\left[\ddot{u}_{i}\right]$ are related by

$$
g_{i j k l}\left[u_{k, l}\right]=\rho\left[\ddot{u}_{i}\right] .
$$

Introducing (2.6) into (2.7), we obtain on the surface (2.4), where $\left[f^{\prime \prime}\right] \neq 0$,

$$
\left(g_{i j k l} \Phi_{, i} \Phi_{, l}-\rho \dot{\Phi}^{2} \delta_{i k}\right) b_{k}=0 .
$$

This equation has a non-trivial solution for $b_{k}$ provided that

$$
\left|g_{i j k l} \Phi_{, j} \Phi_{, l}-\rho \dot{\Phi}^{2} \delta_{i k}\right|=0 .
$$

This is the secular equation for the second-order discontinuity.

Let $n$, be the unit normal to the surface (2.4) and let $V$ be the velocity of the surface. Then

$$
n_{i}=\Phi_{. i}\left(\Phi_{, i} \Phi_{. i}\right)^{-1 / 2} \text { and } V=-\dot{\Phi}\left(\Phi_{, i} \Phi_{, j}\right)^{-1 / 2} .
$$

Let $S(=1 / V)$ denote the slowness and let $S_{i}=S n_{i}$. Then, with (2.10), Eq. (2.8) may be written as

$$
\Lambda_{i k} b_{k}=0,
$$

where

$$
\Lambda_{i k}=g_{i j k l} S_{i} S_{l}-\rho \delta_{i k} .
$$

The secular equation (2.9) then becomes

$$
\Omega\left(S_{i}\right)=\left|\Lambda_{i k}\right|=0
$$

This represents the slowness surface in $S_{i}$-space.

The energy flux vector $R_{i}$ is defined as the rate at which energy leaves the material across an element of area normal to the $x_{i}$-axis, measured per unit area. It is given [1] by

$$
R_{i}=-\sigma_{i i} \dot{u}_{i} .
$$

We consider a time-dependent deformation given by (2.5). Let $t$ be the time at which the surface $\Phi\left(x_{i}, t\right)=0$ passes the point $x_{i}$. We suppose that at times $t-\epsilon_{1}$ and 
$t+\epsilon_{2}$, where $\epsilon_{1}$ and $\epsilon_{2}$ are positive, $u_{i}\left(x_{i}\right)=0$. It follows from (2.5) that

$$
f\left\{\Phi\left(x_{i}, t-\epsilon_{1}\right)\right\}=f\left\{\Phi\left(x_{i}, t+\epsilon_{2}\right)\right\}=0 .
$$

Then, using (2.1) and (2.5), we see that the resultant $R_{i}$ of the energy flux vector is given by

$$
R_{i}=\int_{t-\epsilon_{1}}^{t+\epsilon_{2}} R_{i} d t=-\int_{t-\epsilon_{1}}^{t+\epsilon_{2}} g_{i j k l}\left(\frac{1}{2} b_{k, l} b_{j} \frac{\partial\left(f^{2}\right)}{\partial t}+b_{k} b_{i} f_{, l} \dot{f}\right) d t
$$

Since

$$
f_{, l}=f^{\prime} \Phi, \quad \text { and } \quad \dot{f}=f^{\prime} \dot{\Phi}
$$

we have

$$
f_{, l} \dot{\Phi}=\dot{f} \Phi_{. l}
$$

Using this relation to substitute for $f_{. l}$ in (2.16) and bearing in mind relations (2.15) and (2.10), Eq. (2.16) becomes

$$
Q_{i}=\int_{t-\epsilon_{1}}^{t+\epsilon_{2}} g_{i j k l} b_{k} b_{i} n_{l} S \dot{f}^{2} d t
$$

3. A particular case. We consider a particular case when

$$
g_{i j k l}=\mu\left(\delta_{i k} \delta_{j l}+\delta_{j k} \delta_{i l}\right)+\lambda \delta_{k l} \delta_{i j}+\gamma\left(\delta_{k 1} \delta_{l 2}+\delta_{k 2} \delta_{l 1}\right) \delta_{i j},
$$

where $\mu, \lambda, \gamma$ are constants. Introducing this relation into (2.12), we obtain

$$
\Lambda_{i k}=\left(\mu S^{2}-\rho\right) \delta_{i k}+(\lambda+\mu) S_{\imath} S_{k}+\gamma\left(\delta_{k 1} S_{2}+\delta_{k 2} S_{1}\right) S_{i} .
$$

For a wave propagating in the 1, 2-plane, we may write

$$
S_{1}=S n_{1}, \quad S_{2}=S n_{2}, \quad S_{3}=0 .
$$

With (3.2), this yields

$$
\left\|\Lambda_{i k}\right\|=\left\|\begin{array}{ccc}
{\left[(\lambda+\mu) n_{1}^{2}+\gamma n_{1} n_{2}+\mu\right] S^{2}-\rho,} & {\left[(\lambda+\mu) n_{1} n_{2}+\gamma n_{1}^{2}\right] S^{2},} & 0 \\
{\left[(\lambda+\mu) n_{1} n_{2}+\gamma n_{2}^{2}\right] S^{2},} & {\left[(\lambda+\mu) n_{2}^{2}+\gamma n_{1} n_{2}+\mu\right] S^{2}-\rho,} & 0 \\
0, & 0, & \mu S^{2}-\rho
\end{array}\right\| .
$$

Introducing (3.4) into the secular equation (2.13), we obtain

$$
\left[(\lambda+2 \mu)+2 \gamma n_{1} n_{2}\right] S^{2}-\rho=0 \text { or } \mu S^{2}-\rho=0 .
$$

Similarly, Eq. (2.11) yields

$$
\begin{gathered}
\left\{\left[(\lambda+\mu) n_{1}^{2}+\gamma n_{1} n_{2}+\mu\right] S^{2}-\rho\right\} b_{1}+\left[(\lambda+\mu) n_{1} n_{2}+\gamma n_{1}^{2}\right] S^{2} b_{2}=0, \\
{\left[(\lambda+\mu) n_{1} n_{2}+\gamma n_{2}^{2}\right] S^{2} b_{1}+\left\{\left[(\lambda+\mu) n_{2}^{2}+\gamma n_{1} n_{2}+\mu\right] S^{2}-\rho\right\} b_{2}=0,} \\
\left(\mu S^{2}-\rho\right) b_{3}=0 .
\end{gathered}
$$

For the wave corresponding to the first of Eqs. (3.5), we obtain

$$
b_{1} / b_{2}=n_{1} / n_{2} \text {. }
$$


Thus, the wave is polarized longitudinally.

For this wave, the resultant energy flux vector $R_{i}$ given by (2.19) becomes, with (3.1), (3.7) and $n_{3}=0$,

$$
\mathbb{R}_{i}=\int_{t-\epsilon_{1}}^{t+\epsilon_{2}}\left\{(\lambda+2 \mu)+2 \gamma n_{1} n_{2}\right\} S b^{2} \dot{f}^{2} n_{i} d t,
$$

where $b$ is the magnitude of $b_{i}$. It is evident that provided $n_{i}$ is constant during the time interval $\left(t-\epsilon_{1}, t+\epsilon_{2}\right), \mathbb{R}_{i}$ is parallel to $n_{i}$; i.e., the resultant energy propagation vector is perpendicular to the wave-front. We shall, however, show that it is not, in general, normal to the slowness surface.

The normal to the slowness surface is in the direction $\partial \Omega / \partial S_{i}$, where $\Omega$ is defined by (2.13).

Clearly, from (3.2) the slowness surface $\Omega\left(S_{i}\right)=0$ is symmetric with respect to the plane $S_{3}=0$, and hence the normals to $\Omega\left(S_{i}\right)=0$ at points on $S_{3}=0$ will lie on the plane $S_{3}=0$. The components of the normal to the sheet of $\Omega\left(S_{i}\right)$ corresponding to the wave $(3.5)_{1}$ can be read off from $(3.5)_{1}$. Thus on $S_{3}=0$,

$$
\frac{\partial \Omega}{\partial S_{1}} / \frac{\partial \Omega}{\partial S_{2}}=\left[(\lambda+2 \mu) S_{1}+2 \gamma S_{2}\right] /\left[(\lambda+2 \mu) S_{2}+2 \gamma S_{1}\right], \quad \frac{\partial \Omega}{\partial S_{3}}=0,
$$

and thus at $S_{2}=S_{3}=0$

$$
\frac{\partial \Omega}{\partial S_{1}} / \frac{\partial \Omega}{\partial S_{2}}=(\lambda+2 \mu) / 2 \gamma, \quad \frac{\partial \Omega}{\partial S_{3}}=0 .
$$

Thus, $\partial \Omega / \partial S_{1}$ is plainly not in the direction of the resultant energy propagation vector, as it always is [1] for a linear elastic material for which a strain-energy function exists.

\section{References}

[1] J. A. Schouten, Tensor analysis for physicisis, Clarendon Press, Oxford (1951)

[2] E. Courant and D. Hilbert, Methods of mathematical physics, Vol. II, Interscience, New York (1962)

[3] R. S. Rivlin, An introduction to non-linear continuum theories in mechanics and physics, Edizioni Cremonese, Rome (1970)

[4] A. E. Green and P. M. Naghdi, Arch. Rat. Mech. Anal. 40, 37 (1971) 